\title{
BENEFÍCIOS DAS ATIVIDADES AQUÁTICAS PARA IDOSOS
}

\author{
BENEFITS OF AQUATIC ACTIVITIES FOR SENIORS \\ Luciano Amado Leão ${ }^{a^{*}}$, Gilberto Aparecido Gomes Luciano ${ }^{b^{*}}$, Yasmin Barros de Santana ${ }^{c^{*}}$, \\ Mariana Rotta Bonfim ${ }^{\mathrm{d}^{*}}$
}

styflerrico@gmail.coma ${ }^{a}$,gibagomes1983@gmail.com ${ }^{\mathrm{b}}$, yasminsantana2015@gmail.comc, mariana.bonfim@prof.uscs.edu.br ${ }^{\mathrm{d}}$ Universidade Municipal de São Caetano do Sul - USCS

Data de Submissão 09/04/2019

Data de aceite:21/10/2019

\section{RESUMO}

Introdução: Os profissionais da educação física devem estar sempre atualizados sobre os efeitos da prática de atividades aquáticas na saúde física, mental e emocional dos idosos, visando a melhoria de sua qualidade de vida, condição de saúde e bem-estar. Objetivo: Identificar as mais recentes evidências nacionais sobre os benefícios da prática de atividades aquáticas na vida dos idosos. Métodos: Revisão bibliográfica cujo levantamento dos trabalhos foi realizado nas bases de dados SciELO e LILACS, utilizando as combinações dos termos "Idoso(s)", "atividades aquáticas", "hidroginástica" e "natação", publicados no período de Agosto de 2013 a Setembro de 2018. Resultados: A prática de hidroginástica e de fisioterapia aquática altera positiva e significativamente a performance dos idosos nos testes de equilíbrio e de flexibilidade quando comparados aos sedentários, porém não está associada a bons índices de força muscular. A fisioterapia aquática contribui para a melhora dos quadros de dor e da capacidade funcional na osteoartrite de joelho, e melhorou o desempenho no teste de 6 minutos dos idosos; porém, os praticantes de dança apresentaram melhor desempenho que os praticantes de hidroginástica. Conclusão: As atividades aquáticas de hidroginástica e hidroterapia contribuem para manutenção e melhora da flexibilidade, equilíbrio e capacidade funcional dos idosos; apesar de melhorarem a dor na oesteoartrite de joelho, não estão associadas a bons índices de força muscular de membros inferiores.

Palavras-xhave: Idoso; ambiente aquático; ginástica; natação

\section{ABSTRACT}

Introduction: Physical education professionals should always be updated about the effects of aquatic activities practice in physical health, mental and emotional in the aged, aiming to improve their quality of life, health conditions and well-being. Objective: Identify the most recent national evidences about aquatic activities practice in the aged life. Methods: Bibliographic review which search was performed in SciELO and LILACS databases, using the combination of the terms "aged", "aquatic activities", "water aerobics" and "swimming", published in the period of 2013 August and 2018 September. Results: Water aerobics and hydrotherapy alter positive and significantly the performance in balance and flexibility tests of aged when compared to the sedentary, however there are not associated to better status of muscle force. Hydrotherapy contributes to the improvement of the pain and functional capacity on knee osteoarthritis cases, and improved the performance on 6 minutes test; however, dancer practitioners presented better performance compared to the water aerobics practitioner. Conclusion: Aquatic activities of water aerobics and hydrotherapy contribute to the maintanence and improvement of flexibility, balance and functional capacity of the aged; although improving pain in knee osteoarthritis, they are not associated with better status of lower limbs muscle force.

Keywords: Aged; aquatic environment; gymnastics; swiming 


\section{Introdução}

A população mundial vem apresentando uma tendência de envelhecimento nas últimas décadas, tanto pelo aumento da expectativa de vida devido à melhoria das condições de saúde, como por uma redução da taxa de natalidade. No Brasil, essa parcela da população apresentou um crescimento de $18 \%$ nos últimos cinco anos, passando de 25 milhões para mais de 30 milhões de idosos; atualmente, cerca de $15 \%$ da população é de idosos, dos quais a grande maioria são mulheres ${ }^{1}$.

A definição de idoso como pessoa com mais de 60 anos de idade é estabelecida na legislação brasileira, pela Lei $\mathrm{n}^{\circ} 8.842$, de 4 de janeiro de 1994, regulamentada pelo Decreto $\mathrm{n}^{\circ} 1.948$, de 3 de julho de 1996, sendo reafirmado no Estatuto do Idoso publicado pelo Ministério da Saúde 2 . De acordo com esse documento, o idoso goza de todos os direitos fundamentais inerentes à pessoa humana, sendo a ele asseguradas "todas as oportunidades e facilidades, para preservação de sua saúde física e mental e seu aperfeiçoamento moral, intelectual, espiritual e social, em condições de liberdade e dignidade".

Apesar do aumento nos seus anos de vida, os idosos apresentam uma redução no nível de atividade física regular, acarretando em situações de elevada prevalência de sedentarismo; estes quadros podem estar associados a situações de deficiência física, deficiência mental e exclusão social, podendo evidenciar alguns sinais de uma possível depressão e de doenças cardiovasculares ${ }^{3}$. De acordo com Gobbi, Villar, Zago ${ }^{4}$, a prática de atividade física, como uma leve caminhada ou até mesmo a execução de atividades domésticas em geral, pode melhorar a aptidão física e também a capacidade funcional destes indivíduos, contribuindo significativamente para a manutenção de sua independência e autonomia.

Existem várias possibilidades de atividades físicas para idosos, porém verifica-se que o mais comum dentre essas faixas etárias é a prática de atividades em meio aquático, pois são consideradas práticas de baixo impacto, o que evitaria a ocorrência de lesões articulares ${ }^{5}$. Ademais, há evidências de que este tipo de atividades está associado a melhoras no condicionamento físico, resistência e força muscular, capacidade pulmonar, frequência cardíaca, mobilidade articular, postura e performance nas atividades diárias de idosos ${ }^{6,7}$.

Esta realidade evidencia a necessidade de nós, enquanto profissionais da educação física, estarmos atentos e atualizados sobre os efeitos da prática de atividades aquáticas na saúde física, mental e emocional dos idosos, visando a melhoria de sua qualidade de vida, condição de saúde e bem-estar. Nesse sentido, o objetivo da presente pesquisa será identificar as mais recentes evidências nacionais sobre os benefícios da prática de atividades aquáticas na vida dos idosos.

\section{Métodos}

O presente trabalho se caracteriza por uma revisão bibliográfica, sendo que o levantamento dos trabalhos para essa pesquisa foi realizado nas bases de dados Scientific Library Online (SCIELO) e Literatura Latino Americana e do Caribe em Ciências da Saúde (LILACS), utilizando as combinações do descritor "Idoso(s)", com as palavras-chave "atividades aquáticas", "hidroginástica" e "natação", publicados no período de Agosto de 2013 a Setembro de 2018.

Foram selecionados para este trabalho apenas artigos originais, publicados em língua portuguesa e inglesa, os quais aplicaram atividades físicas no ambiente aquático para idosos, a fim de identificar quais seus efeitos nos âmbitos físico, psíquico, de saúde e de qualidade de vida para essa faixa etária. Foram excluídos artigos de revisão, editorias, cartas e trabalhos acadêmicos.

Dos artigos selecionados foram extraídas as informações sobre o objetivo do trabalho, a amostra (tamanho amostral, sexo, idade, grupos de estudo), os dados das intervenções propostas (tempo de exposição e tipo de atividade), as ferramentas de análise, os resultados encontrados e as conclusões dos estudos.

Com relação aos resultados dos artigos pesquisados por diversos bancos de dados, tais como Scielo, Lillacs e Periódicos Capes, pode se obsevar na figura abaixo que somente o banco de dados Scielo não foram encontrados resultados com êxito. Entretanto foram realizadas buscas de cinco e dez anos atrás. 


\section{Resultados}

Dos trabalhos analisados, observou-se que 06 deles avaliaram os efeitos da hidroginástica nos idosos, e os outros 03 avaliam os efeitos da hidroterapia administrada por fisioterapeutas; optamos por inserir esses artigos, pois a estrutura das aulas e as atividades propostas são semelhantes àquelas da hidroginástica. Os artigos sobre hidroginástica avaliaram seus efeitos no equilíbrio corporal, na força de membros inferiores e no desempenho ocupacional de idosos, bem como compararam seus benefícios aos da dança ou do treinamento combinado na capacidade funcional e flexibilidade; os trabalhos de fisioterapia aquática, por sua vez, avaliaram prioritariamente seus efeitos no equilíbrio corporal, capacidade funcional e capacidade respiratória dos idosos (tabela 1).

No que diz respeito aos efeitos no equilíbrio, verificou-se que a prática de 60 minutos de hidroginástica, com ênfase nos exercícios de deslocamento (correr, andar) e exercícios localizados, realizados $2 \mathrm{x}$ vezes por semana por 3 meses, altera positiva e significativamente os índices de equilíbrio postural ${ }^{8}$, sendo que sua prática por mais de 6 meses acarretou em melhor performance dos idosos nos testes de equilíbrio quando comparados aos sedentários?.

Os trabalhos de fisioterapia aquática, desenvolvidos em sessões de 40-50 minutos, 1 a 3 vezes por semana, por 08-09 semanas, identificaram melhoras significantes nesta variável apenas nas intervenções que utilizaram exercícios de equilíbrio ${ }^{10,-12}$; estas intervenções de fisioterapia aquática também foram eficientes em melhorar o desempenho no teste de 6 minutos dos idosos ${ }^{10-12}$.

Todavia, verificou-se que praticantes de 3 sessões semanais de 45 minutos de hidroginástica apresentaram valores inferiores de distância percorrida no teste de 6 minutos quando comparados a praticantes de 3 sessões semanais de 45 minutos de dança ${ }^{13}$. Ademais, quando comparada a outras práticas de atividades físicas, verificou-se que a flexibilidade de idosos praticantes de 2-3 sessões semanais de 50 minutos de hidroginástica não apresenta diferenças significantes àquela de idosos praticantes de 2-3 sessões semanais de 40 minutos de treinamento combinado (aeróbio e resistido), porém ambos os grupos apresentavam valores superiores aos idosos sedentários ${ }^{14}$.

No que diz respeito à força muscular de membros inferiores em idosas praticantes de hidroginástica, verificou-se que apenas $6 \%$ delas apresentavam nível de força muito bom, sendo que a grande maioria apresentou nível fraco, fato que apresentou relação com a má funcionalidade do joelho ${ }^{15}$. Todavia, verificou-se que a oferta de fisioterapia aquática em duas sessões semanais de 50 minutos, por 09 semanas, para idosos com osteoartrite no joelho, foi eficiente em melhorar os quadros de dor e sua capacidade funcional ${ }^{11}$.

Apesar de todos esses pontos positivos como consequência da prática da hidroginástica, o que se percebe entre os idosos praticantes desta modalidade de exercícios é o relato de problemas quanto ao desempenho ocupacional; esta variável está relacionada às atividades cotidianas que os idosos entendem ser mais importantes no diaa-dia, mas que não conseguem realizar. Dentre todas as áreas ocupacionais, os idosos relataram que o lazer é o maior problema, sendo a prática de exercício físico no lazer a maior dificuldade; eles relataram que além da indicação médica ser restrita a esse tipo de atividade, a oferta de atividades para esse público, principalmente em projetos de extensão de faculdades, privilegia esse tipo de prática ${ }^{16}$.

\section{Discussão}

O público idoso, de maneira geral, apresenta como consequência da idade uma série de sintomas que dificultam a prática de exercícios em solo, como por exemplo os episódios de dores e doenças articulares, além da fraqueza muscular e a redução do equilíbrio, fatores que estão diretamente associados a desordens de marcha. Adversamente, o ambiente aquático tem demonstrado ser um aliado no aumento do nível de atividade física desta população, uma vez que ele contribui para a menor sobrecarga nas articulações, reduzindo assim os riscos de lesões e de quedas; ademais, o meio líquido proporciona 
o estado da flutuação, o que permite a realização de ações e movimentos que dificilmente seriam praticados em solo ${ }^{17}$.

De fato, o que se observou nesse levantamento da literatura é que a prática de hidroginástica e de fisioterapia aquática altera positiva $e$ significativamente a performance dos idosos nos testes de equilíbrio e de flexibilidade quando comparados aos sedentários; porém, estas práticas não se associaram a bons índices de força muscular. Vale ressaltar que a fisioterapia aquática contribuiu para a melhora dos quadros de dor e da capacidade funcional na osteoartrite de joelho, além de melhorar o desempenho no teste de 6 minutos dos idosos; porém, os praticantes de dança apresentaram melhor desempenho que os praticantes de hidroginástica nesse teste.

No que diz respeito à flexibilidade e ao equilíbrio, os trabalhos têm demonstrado que a prática de atividades aquáticas está diretamente associada à melhora destas variáveis, realidade extremamente relevante na vida dos idosos. Isso porque, ambas as capacidades físicas supracitadas estão relacionadas à saúde da população, sendo que a manutenção ou melhoria de seus níveis pode refletir em melhores desempenhos na realização das atividades de vida diária, além de contribuir na redução do risco de quedas ${ }^{18}$. Outra capacidade física relacionada às quedas é a força muscular, visto que maiores níveis de força contribuem na redução de sua ocorrência; a esse respeito, tem-se observado que por apresentarem uma tendência à menor porcentagem de massa magra $\mathrm{e}$, consequentemente, menor força, as mulheres idosas geralmente estão mais expostas às quedas ${ }^{19}$.

De maneira geral, o envelhecimento está associado à diminuição da função muscular, sendo esta redução mais evidente a partir dos 60 anos, principalmente no público feminino. O que se observa é que a força muscular máxima é alcançada até os 30 anos, e até mais ou menos os 50 anos se mantém estável, até que então ela começa a apresentar um decréscimo; entre os 50 e 70 anos ocorre uma perca de cerca de $15 \%$ a cada dez anos, após isso essa diminuição aumenta para $30 \%$ e mantém reduzindo a cada década ${ }^{20}$.

Como verificado neste estudo, a hidroginástica parece não ser a prática mais eficaz para aumento da força e da massa muscular de membros inferiores. Está bem descrito na literatura que um treinamento específico de força como a musculação, por exemplo, gera ganhos mais significativos devido à sua especificidade, em comparação à hidroginástica. Todavia, ressalta-se que a hidroginástica está associada ao aumento no tônus muscular, porém em menor proporção àquele causado pelo treino de força, estando ainda associado a um melhor sem riscos de lesão, uma vez que a água possui uma densidade maior, gerando menores impactos ${ }^{15}$.

Além da menor densidade, a água tem outras propriedades físicas como pressão hidrostática e empuxo, que permitem vários exercícios terapêuticos sejam realizados em uma condição de baixo impacto articular e redução. De fato, os resultados de Souza, Moura, Bastone ${ }^{11}$ mostram que após a prática de atividade aquática houve melhora significativa para quem tem a osteoartrite (OA) de joelho, um quadro altamente prevalente na população idosa relacionado à perda de função e mobilidade dos indivíduos. Tais atividades físicas como a hidroginástica ou hidroterapia traz propriedades físicas da água, como densidade.

Vale ressaltar que além destas propriedades físicas da água, as práticas aquáticas são realizadas em ambiente aquecido e, portanto, a água desempenha ainda um papel importante na analgesia, na redução da rigidez e na redução de impacto articular, favorecendo a realização de exercícios por pessoas que apresentam limitações articulares, como a osteoartrite (OA) de joelho, que é uma doença crônico-degenerativa caracterizada pelo desgaste da cartilagem articular.

Estas características da água contribuem para aumentar o nível de atividade física dos idosos, o que é extremamente importante. Estudos mostram que o envelhecimento associado ao sedentarismo gera mudanças não apenas no sistema neuromuscular, mas também no somatossensorial, vestibular e visual; assim, o exercício físico é importante na manutenção da funcionalidade dessa população. As atividades aquáticas estudadas nesta pesquisa indicam que sua prática traz benefícios e melhoria da capacidade aeróbia, capacidade funcional, equilíbrio postural e flexibilidade dos idosos, contribuindo para a manutenção e melhoria de sua saúde.Sabese, portanto, que a água permite a imersão e a 
flutuação corporal, facilitando a reprodução de movimentos compostos de maneira segura e variada, minimizando os impactos (quando comparados com os exercícios no solo), o que pode permitir o trabalho de mobilidade corporal e de flexibilidade de forma segura e gradual, não havendo contraindicações para sua prática ${ }^{21}$. Estas características acabam influenciando a classe médica que, por sua vez, recomendam quase com exclusividade a hidroginástica para a população idosa.

De fato, a maioria dos idosos procura a hidroginástica por recomendação médica, e que acabam aceitando essa prática pois a execução dos movimentos se torna fácil. Todavia, observouse nesse estudo que alguns idosos se queixam de ter essa modalidade como praticamente a única atividade física prescrita pelos médicos. Nessa perspectiva, ressaltamos que além da ilegalidade do exercício da profissão médica, uma vez que a indicação e prescrição de exercícios devem ser feitas pelo professor de Educação Física, esta visão está equivocada, pois o idoso pode e deve praticar qualquer atividade que o faça se sentir bem consigo mesmo, desde que bem orientado por um bom profissional e respeitando suas limitações.

Em suma, pode-se concluir que a prática regular e orientada de hidroginástica traz inúmeros benefícios no para os idosos, como melhora da capacidade aeróbia, da capacidade funcional, do equilíbrio postural, e da flexibilidade, auxiliando no controle de doenças crônicas como a artrose, e promovendo a inserção deste indivíduo no meio social, propiciando ao mesmo tempo uma melhor qualidade de vida e uma sensação de pertencimento a um grupo.

\section{Conclusão}

As atividades aquáticas de hidroginástica e hidroterapia contribuem para manutenção e melhora da flexibilidade, equilíbrio e capacidade funcional dos idosos; apesar de melhorarem a dor na oesteoartrite de joelho, não estão associadas a bons índices de força muscular de membros inferiores.

\section{Referências}

1. IBGE. Instituto Brasileiro de Geografia e Estatística. Número de idosos cresce $18 \%$ em 5 anos e ultrapassa 30 milhões em 2017. Disponível em: <https:// agenciadenoticias.ibge.gov.br/agencia-noticias/2012agencia-de-noticias/noticias/20980-numero-deidosos-cresce-18-em-5-anos-e-ultrapassa-30-milhoesem-2017>. Acesso em: 14 Set. 2018.

2. Brasil. Ministério da Saúde. Estatuto do idoso. Brasília: Editora do Ministério da Saúde, 2009.

3. Camarano AA. Envelhecimento da população brasileira: uma contribuição demográfica. Rio de Janeiro, IPEA ; 2002.

4. Gobbi S, Villar R, Zago AS. Bases Teórico-Práticas do Condicionamento Físico. Rio de Janeiro: Guanabara, 2005.

5. Simões RR, Portes Junior M, Moreira WW. Idosos e hidroginástica: corporeidade e vida. Revista Brasileira de Ciência e Movimento, Brasília .2011; 19(4): 40-50.

6. Ortega RF. Quais os fatores de adesão num programa de atividades aquáticas para a terceira idade para uma melhora na qualidade de vida. Revista Didática Sistêmica, Rio Grande, edição especial.2010 ; 32-45.

7. Vendana TA, Santos RN , Pereira JM , Araujo SP , Portes Junior M , Portes LA. Influência da hidroginástica sobre a composição corporal, aspectos cardiovasculares, hematológicos, função pulmonar e aptidão física de adultos e idosos. Brazilian Journal of Biomotricity, Itaperuna. $2011 ; 5(2)$ : 65-79.

8. Souza Junior RO, Deprá PP, Silveira AM . Efeitos Da hidroginástica com exercícios dinâmico em deslocamento sobre o equilíbrio corporal de idosos. Revista Fisioterapia e Pesquisa. 2017; 24(3): 303-310.

9. Silva GR, Terra GDSV, Tavares MR, Neiva CM , Rodrigues CAC , Martins DW et al . Idosos praticantes e não praticantes de exercícios físicos : uma comparação do estado de equilíbrio . Revista Kairós gerontologia. 2015; 18(2): 311-326.

10. Motta LRS, Seero AR, Machado EC, Mello NF, Filippin NT , Gobbato RE et al . Avaliação do equilíbrio e do condicionamento cardiorrespiratório de participantes do grupo de atividades hidrocinesioterapeuticos do centro universitário Franciscano em Santa Maria RS . Estudos Interdisciplinares do envelhecimento. 2015;20(3):745754.

11. Souza AA, Moura JÁ, Bastone AC. Efetividade de um programa de fisioterapia aquática na capacidade aeróbia , dor, rigidez , equilíbrio e função física de idosos com osteoartrite de joeho . Fisioterapia Brasil. 2017;18(2):165171. 
12. Siqueira AF, Rebesco DB, Amaral FA, Maganhini CB , Agnol SMD , Furmann $\mathrm{M}$ et al . Efeito de um programa de fisioterapia aquática no equilíbrio e capacidade funcional de idosos. Saúde e Pesquisa. 2017;10(2):331-338.

13. Albuquerque IM, Emmanouilidis A, Ortolan T, Cardoso DM , Gass R, Jost RT et al. Capacidade funcional submáxima e força muscular respiratória entre idosos praticantes de hidroginástica e dança : um estudo comparativo . Revista Brasileira de Geriatria e Gerontologia. 2013;16(2):327-336.

14. Zambon TB, Gonelli PRG, Gonçalves RD, Borges BLA , Montebelo MIL, Cesar MC . Análise comparativa da flexibilidade de mulheres idosas ativas e não ativas . Revista Acta Fisiátrica. 2015; 22(1): 14-18.

15. Oliveira DV, Santos AT, Antunes MD, Nascimento Junior JRA , Bertolini SMMG . Força muscular e funcionalidade do joelho de idosas praticantes de hidroginástica . Revista Cogitare Enfermagem. 2017; 2(22): 1-9.
16. Estivalet KM, Corazza ST. Desempenho ocupacional de idosos praticantes de hidroginástica. Cadernos Brasileiros de Terapia Ocupacional. 2017; 25(2):315-323.

17. Booth CE. Water exercise and its effects on balance and gait to reduce the risk of falling in older adults. Activities, Adaptation Aging. 2004;28(4):45-57.

18. Resende SM, Rassi CM,Viana FP. Efeitos da hidroterapia na recuperação do equilíbrio e prevenção de quedas em idosas. Rev Bras Fisioter. 2008;12(1):57-63.

19. Perracini MR, Ramos LR. Fatores associados a quedas em uma coorte de idosos residentes na comunidade. Rev Saúde Pública. 2002;36(6):709-16.

20. Lima RM, Ferreira CES, Bezerra LMA, Rabelo HT, da Silva Junior C, dos Santos EP, et al. Estudo de associação entre força muscular e massa magra em mulheres idosas. Rev. Bras. Ciênc. Esporte. [Internet] 2012;34(4) [acesso em 04 nov 2016]. Disponível: http://dx.doi.org/10.1590/ S0101-32892012000400013.

21. Bastos CC, Oliveira EM. Sindrome da fibromialgia: Tratamento em piscina aquecida. Lato\&Sensu,Belém .2003;4(1): 3-5 .

Quadro 1. Apresentação dos trabalhos analisados.

\begin{tabular}{|c|c|c|c|c|c|c|}
\hline \multirow[b]{2}{*}{ REFERÊNCIA } & \multirow[b]{2}{*}{ OBJETIVO } & \multicolumn{3}{|c|}{ METODOLOGIA } & \multirow[b]{2}{*}{ RESULTADOS } & \multirow[b]{2}{*}{ CONCLUSÃO } \\
\hline & & AMOSTRA & $\begin{array}{l}\text { ATIVIDADES } \\
\text { PROPOSTAS }\end{array}$ & $\begin{array}{l}\text { VARIÁVEIS } \\
\text { AVALIADAS }\end{array}$ & & \\
\hline $\begin{array}{l}\text { Zambon TB, } \\
\text { Gonelli PRG, } \\
\text { Gonçalves RD, et } \\
\text { al. Revista Acta } \\
\text { Fisiátrica. 2015; } \\
\text { 22(1): 14-18. }\end{array}$ & $\begin{array}{c}\text { Comparar a } \\
\text { flexibilidade de } \\
\text { mulheres idosas } \\
\text { praticantes de } \\
\text { hidroginástica, } \\
\text { treinamento } \\
\text { combinado e não } \\
\text { ativas. }\end{array}$ & $\begin{array}{c}\text { Estudo } \\
\text { observacional e } \\
\text { transversal. } \\
\mathrm{N}=60 \text { } \text {, , entre } 60 \text { e } \\
80 \text { anos, agrupadas } \\
\text { em: } \\
\text { Hidroginástica } \\
\text { (HIDRO) (n=20), } \\
\text { Treinamento } \\
\text { Combinado (TC) } \\
\text { (n=20), Não ativas } \\
\text { (NA) (n=20) }\end{array}$ & $\begin{array}{l}\text { HIDRO: } 02-03 \mathrm{x} / \mathrm{sem}, \\
50 \text { ' (10' aquecimento, } \\
30 \text { ' parte principal, } 10 \text { ' } \\
\text { volta á calma) } \\
\text { TC: } 02-03 \mathrm{x} / \mathrm{sem}, 60 \text { ' } \\
\text { (3'-5' alongamento, } 15 \text { ' } \\
\text { aeróbio, } 15 \text { ' ex. força } \\
\text { muscular, } 15 \text { ' aeróbio, } \\
\text { 10' volta a calma) } \\
\text { NA: Sem atividade }\end{array}$ & $\begin{array}{l}\text { Nível de Atividade } \\
\text { Física: IPAC } \\
\text { Medidas } \\
\text { antropométricas } \\
\text { Flexibilidade: teste } \\
\text { de schober, teste de } \\
\text { sentar e alcançar, } \\
\text { goniometria de } \\
\text { flexão e extensão de } \\
\text { quadril }\end{array}$ & $\begin{array}{c}\text { Sem diferença nas } \\
\text { medidas antropométricas } \\
\text { > Extensão e flexão de } \\
\text { quadril nas ativas, sem } \\
\text { diferença entre HIDRO } \\
\text { e TC }\end{array}$ & $\begin{array}{c}\text { Treinamento de } \\
\text { HIDRO e TC } \\
\text { proporcionaram } \\
\text { melhora na flexão e } \\
\text { extensão de quadril } \\
\text { de idosas. }\end{array}$ \\
\hline $\begin{array}{l}\text { Junior ROS, } \\
\text { Deprá PP, Silveira } \\
\text { AM. Revista } \\
\text { Fisioterapia e } \\
\text { Pesquisa. 2017; } \\
\text { 24(3): 303-310. }\end{array}$ & $\begin{array}{c}\text { Investigar os efeitos } \\
\text { de um programa de } \\
\text { hidroginástica com } \\
\text { ênfase em exercícios } \\
\text { dinâmicos em } \\
\text { deslocamento sobre } \\
\text { o equilíbrio corporal } \\
\text { de idosos. }\end{array}$ & $\begin{array}{c}\text { Estudo quase } \\
\text { experimental } \\
\text { Grupo } \\
\text { Hidroginástica } \\
\text { (HIDRO): } \mathrm{n}=27, \\
(67,33 \pm 5,53 \text { anos) } \\
\text { Grupo Controle } \\
\text { (GC): } \mathrm{n}=10 \\
(67,74 \pm 7,24 \text { anos })\end{array}$ & $\begin{array}{l}\text { HIDRO: } 02 \mathrm{x} / \mathrm{sem}, 16 \\
\text { semanas, } 50 \text { ' de aula } \\
\text { (10' aquecimento, } \\
\text { 30' parte principal, } \\
\text { 10' volta à calma/ } \\
\text { alongamento) } \\
\text { Ênfase em exercícios } \\
\text { de deslocamento } \\
\text { (andar, correr) e } \\
\text { exercícios localizados }\end{array}$ & $\begin{array}{l}\text { Variáveis do centro } \\
\text { de pressão (COP): } \\
\text { amplitude médio- } \\
\text { lateral, amplitude } \\
\text { ântero-posterior, } \\
\text { deslocamento total } \\
\text { e área do COP }\end{array}$ & $\begin{array}{c}\downarrow \text { redução do } \\
\text { deslocamento total e } \rightarrow \\
\text { das amplitudes ântero- } \\
\text { posterior e da área do } \\
\text { COP na condição olhos } \\
\text { fechados do HIDRO } \\
\uparrow \text { da área do COP do GC }\end{array}$ & $\begin{array}{c}\text { A a prática da } \\
\text { hidroginástica, } \\
\text { enfatizando } \\
\text { exercícios com } \\
\text { diferentes formas } \\
\text { de deslocamentos, } \\
\text { pode alterar } \\
\text { significativamente os } \\
\text { indices de equilíbrio } \\
\text { postural. }\end{array}$ \\
\hline
\end{tabular}




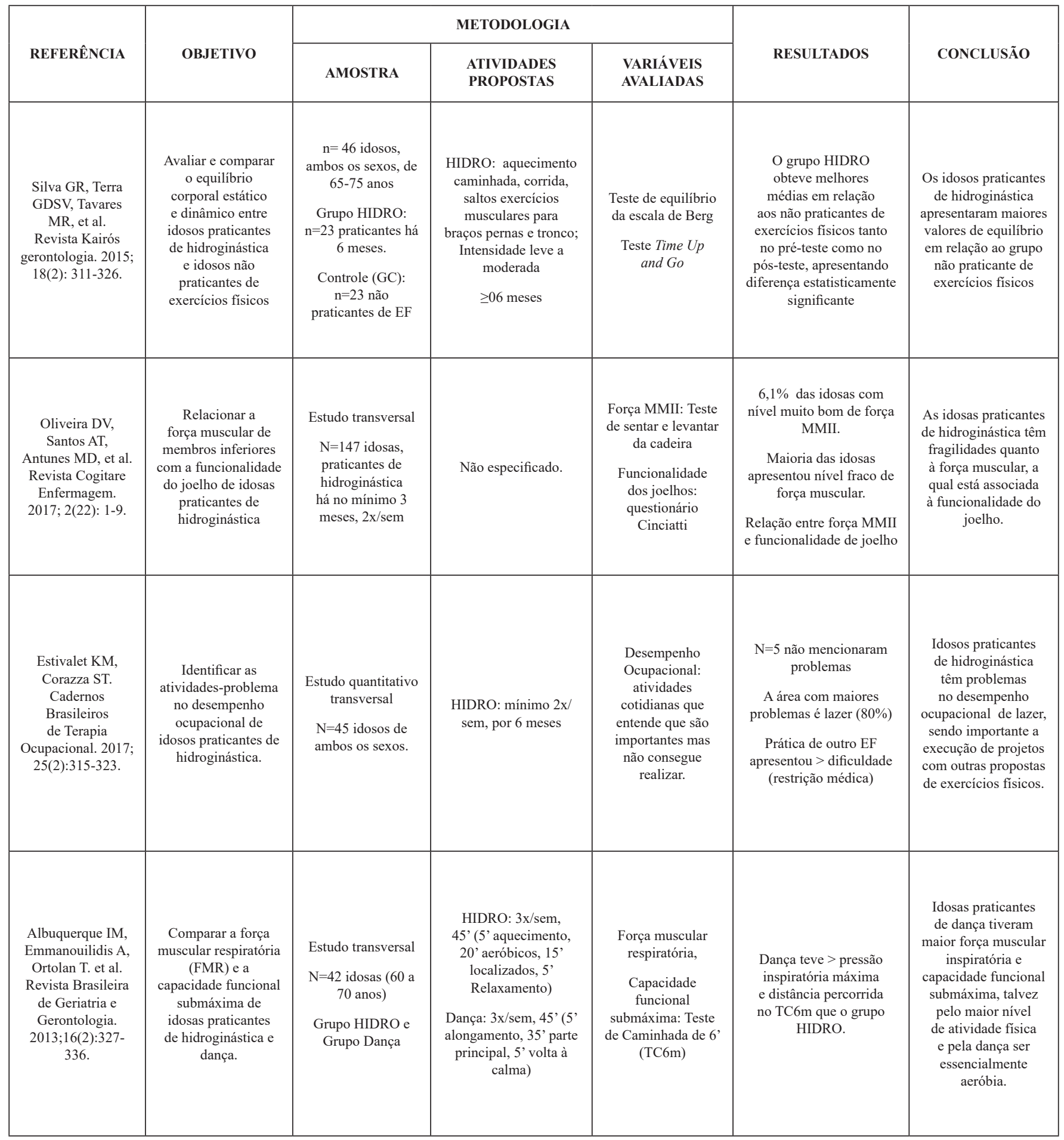




\begin{tabular}{|c|c|c|c|c|c|c|}
\hline \multirow{2}{*}{ REFERÊNCIA } & \multirow{2}{*}{ OBJETIVO } & \multicolumn{3}{|c|}{ METODOLOGIA } & \multirow{2}{*}{ RESULTADOS } & \multirow{2}{*}{ CONCLUSÃO } \\
\hline & & AMOSTRA & $\begin{array}{l}\text { ATIVIDADES } \\
\text { PROPOSTAS }\end{array}$ & $\begin{array}{l}\text { VARIÁVEIS } \\
\text { AVALIADAS }\end{array}$ & & \\
\hline $\begin{array}{c}\text { Motta LRS, Seero } \\
\text { AR, Machado } \\
\text { EC, Mello NF } \\
\text { et al. Estudos } \\
\text { Interdisciplinares } \\
\text { do envelhecimento. } \\
\text { 2015;20(3):745- } \\
754 .\end{array}$ & $\begin{array}{l}\text { Avaliar o equilíbrio } \\
\text { e o condicionamento } \\
\text { cardiorrespiratório } \\
\text { de participantes do } \\
\text { grupo de atividades } \\
\text { de fisioterapia } \\
\text { aquática. }\end{array}$ & $\begin{array}{c}\text { Estudo } \\
\text { experimental } \\
\mathrm{N}=23 \text { idosos }\end{array}$ & $\begin{array}{l}\text { 1x/sem, 50' (10' } \\
\text { aquecimento; } 20 \text { ' } \\
\text { fortalecimento, } \\
\text { equilíbrio e } \\
\text { coordenação; } 10^{\prime} \\
\text { alongamento; 10' } \\
\text { relaxamento), } 08 \\
\text { semanas }\end{array}$ & $\begin{array}{l}\text { Escala de equilíbrio } \\
\text { de Berg } \\
\text { Teste de caminhada } \\
\text { de } 6 \text { minutos } \\
3 \text { momentos: } \\
\text { Inicial, após } 4 \\
\text { semanas, e após } 8 \\
\text { semanas }\end{array}$ & $\begin{array}{l}\uparrow \text { distância percorrida no } \\
\text { TC6m } \\
\text { Melhor desempenho nas } \\
\text { tarefas de equilíbrio }\end{array}$ & $\begin{array}{c}\text { A fisioterapia } \\
\text { aquática tem papel } \\
\text { importante na } \\
\text { manutenção do } \\
\text { equilíbrio e do } \\
\text { condicionamento } \\
\text { cardiorrespiratório de } \\
\text { idosos. }\end{array}$ \\
\hline $\begin{array}{c}\text { Siqueira AF, } \\
\text { Rebesco DB, } \\
\text { Amaral FA et al. } \\
\text { Saúde e Pesquisa. } \\
\text { 2017; } 10(2): 331- \\
\text { 338. }\end{array}$ & $\begin{array}{l}\text { Avaliar o efeito de } \\
\text { um programa de } \\
\text { fisioterapia aquática } \\
\text { para o equilíbrio e } \\
\text { capacidade funcional } \\
\text { de idosos. }\end{array}$ & $\begin{array}{l}\text { Estudo clínico } \\
\text { prospectivo } \\
\mathrm{N}=11 \text { idosos } \\
\text { diabéticos }\end{array}$ & $\begin{array}{c}3 \mathrm{x} / \mathrm{sem}, 40 \text { ' } \\
\text { (aquecimento, } \\
\text { fortalecimento MMSS } \\
\text { e II, equilíbrio, } \\
\text { exercícios aeróbios e } \\
\text { alongamento), } 2 \text { meses }\end{array}$ & $\begin{array}{l}\text { Teste de Equilíbrio } \\
\text { de Tinetti } \\
\text { Teste de caminhada } \\
\text { de } 6 \text { minutos }\end{array}$ & $\begin{array}{c}\text { Melhora significante no } \\
\text { equilíbrio } \\
\uparrow \text { não significante da } \\
\text { distância percorrida }\end{array}$ & $\begin{array}{l}\text { A fisioterapia } \\
\text { aquática melhorou } \\
\text { o equilíbrio mas } \\
\text { não alterou de } \\
\text { forma significante a } \\
\text { resistência aeróbia. }\end{array}$ \\
\hline $\begin{array}{c}\text { Souza AA, Moura } \\
\text { JÁ, Bastone AC. } \\
\text { Fisioterapia Brasil. } \\
\text { 2017;18(2):165- } \\
171 .\end{array}$ & $\begin{array}{l}\text { Avaliar a efetividade } \\
\text { de um programa de } \\
\text { fisioterapia aquática } \\
\text { na capacidade } \\
\text { aeróbia, dor, rigidez, } \\
\text { equilíbrio e função } \\
\text { física de idosos } \\
\text { com osteoartrite no } \\
\text { joelho. }\end{array}$ & $\begin{array}{c}\text { Estudo } \\
\text { intervencionista } \\
\mathrm{N}=10 \text { idosos com } \\
\text { osteoartrite no } \\
\text { joelho }\end{array}$ & $\begin{array}{l}\text { 2x/sem, 50' (20' } \\
\text { aeróbio, 15' } \\
\text { fortalecimento, 10' } \\
\text { alongamento e 5' } \\
\text { relaxamento), } 9 \\
\text { semanas }\end{array}$ & $\begin{array}{c}\text { Questionário } \\
\text { Western Ontario } \\
\text { and McMaster } \\
\text { Universities } \\
\text { Arthritis Index } \\
\text { Teste de caminhada } \\
\text { de } 6 \text { minutos, } \\
\text { sentar e levantar } \\
\text { da cadeira, one leg } \\
\text { stance }\end{array}$ & $\begin{array}{c}\uparrow \text { capacidade aeróbia e } \\
\text { capacidade funcional } \\
\downarrow \text { dor, associada à } \\
\text { melhoria da capacidade } \\
\text { funcional }\end{array}$ & $\begin{array}{c}\text { A fisioterapia } \\
\text { aquática é um } \\
\text { importante recurso } \\
\text { para melhorar a dor, a } \\
\text { capacidade funcional } \\
\text { e a capacidade } \\
\text { aeróbia de idosos } \\
\text { com osteoartrite no } \\
\text { joelho. }\end{array}$ \\
\hline
\end{tabular}

Legenda: $\mathrm{N}=$ número de participantes, $\widehat{\partial}=$ gênero masculino, $q=$ gênero feminino, Hidro = hidroginástica, $\mathrm{TC}=$ treinamento combinado, $\mathrm{Na}=$ não ativas, $\mathrm{GC}=$ grupo controle, $\mathrm{COP}=$ centro de pressão, $\uparrow=$ aumento, $\rightarrow=$ manutenção, $\downarrow=$ redução.

\section{Como citar este artigo:}

Leão LA, Luciano GAG, Santana YB, Bonfim MR. Benefícios das atividades aquáticas para idosos. Rev. Aten. Saúde. 2019; 17(61): 127-134. 